\title{
Advancements in solar neutrino physics
}

\author{
VITO ANTONELLI, LINO MIRAMONTI \\ Physics Department of Milano University and INFN \\ via Celoria 16, Milano, Italia I-20133.
}

June 20, 2021

\begin{abstract}
We review the results of solar neutrino physics, with particular attention to the data obtained and the analyses performed in the last decades, which were determinant to solve the Solar Neutrino Problem, proving that neutrinos are massive and oscillating particles and contributing to refine the Solar Models. We discuss also the perspectives of the presently running experiments in this sector and of the ones planned for the near future and the impact they can have on elementary particle physics and astrophysics.
\end{abstract}

\section{Introduction}

Solar neutrinos study has given, since ever, a fundamental contribution to elementary particle physics and astrophysics, creating a link between these two disciplines. The original idea of Bahcall and Davis to use neutrinos as probes to investigate the Sun's properties opened half a century ago an unexpected scenario, giving rise to the long standing "Solar Neutrino Problem", which had a great impact on our knowledge of particle physics. The answer to this puzzle, found around the turn of the millennium combining the data obtained by the radiochemical experiments, by SuperKamiokande and more recently by SNO and the reactor experiment KamLAND, proved in a crystal clear way that neutrinos are massive and oscillating particles, showing the need to go beyond the original version of the Standard Model and offering a significant test for every theory "beyond the Standard Model".

In the last years a change of paradigm took place in neutrino physics and the attention is now mainly focused on appearance experiments and on the study of artificial sources (short and long baseline reactor and accelerator experiments and superbeams), which are expected to find at least partial answers to the many open questions of neutrino physics. However, solar neutrino physics still can contribute significantly to the search of some of these answers. In particular it can help to improve and complete the knowledge of mass and mixing pattern, to 
study, for the first time with real time experiments, the low energy part of solar neutrino spectrum and look for possible anomalies in the oscillation pattern. The first step in this direction has been moved by the Borexino collaboration and further contributions are expected by this experiment and also by $\mathrm{SNO}+$ and other experiments planned for the near and far future.

The huge quantity of data accumulated and the accuracy reached by many of the different solar neutrino experiments brought back to new life the original purpose of solar neutrino studies, that is the analysis of the mechanisms regulating the Sun's shining and evolution. In particular it could be important to contribute to solve the so called solar metallicity problem, even if this will be a hard task that requires a simultaneous study not only of the pp-chain, but also of the almost unexplored $C N O$ bi-cycle.

In this paper, after a brief review of the milestones of solar neutrino history, with particular attention to the results of the last 10-15 years, we discuss the status of solar neutrino mixing and oscillation in the three flavor scenario and comment on possible future perspectives of this sector.

\section{From the rise of the Solar Neutrino Problem to its solution}

The history of solar neutrino studies started in the latest 1960 in South Dakota, when the first experiment to detect solar neutrinos was built in the Homestake gold mine [1] 2] 3] 4]. It operated continuously from 1970 until 1994, with a large detector consisting in a tank filled with 615 metric tons of liquid perchloroethylene $\mathrm{C}_{2} \mathrm{Cl}_{4}$, suitable to detect ${ }^{7} \mathrm{Be}$ and ${ }^{8} \mathrm{~B}$, and a small signal from the $C N O$ and pep solar neutrinos, via the reaction $\nu_{e}+{ }^{37} \mathrm{Cl} \rightarrow{ }^{37} \mathrm{Ar}+e^{-}$with an energy threshold of $E_{t h}=814 \mathrm{keV}$. The number of detected neutrinos was only about $1 / 3$ of the quantity expected from the Solar Standard Model (SSM) and this large discrepancy is the essence of the Solar Neutrino Problem (SNP). In the following years SSM passed all tests that helioseismology (the science that studies the interior of the Sun by looking at its vibration modes) started to provide and this indicated only two possible solutions to the SNP: Homestake could be wrong, (i.e. the detector was inefficient) or something happened to the neutrinos while travelling from the core of the Sun to Earth where they were detected.

In 1982-83 Kamiokande a large water Čerenkov detector (of 3048 metric tons) was built in Japan [5. It was the first example of real time solar neutrino detectors, which study the Cerenkov light produced by the electrons scattered by an impinging neutrino via the reaction $\nu_{x}+e^{-} \rightarrow \nu_{x}+e^{-}$and by observing the direction of the recoiled neutrino can reconstruct also the incoming neutrino direction. The energy threshold of the reaction is $E_{t h}=7.5 \mathrm{MeV}$ and, therefore, only ${ }^{8} \mathrm{~B}$ and hep neutrinos were detected. At the beginning of the '90s a larger version of the detector was built, Super-Kamiokande, where the active mass was more than 50000 metric tons of pure water, and the energy threshold was 
lowered to [6] $E_{t h}=5.5 \mathrm{MeV}$. The number of detected neutrinos was about $1 / 2$ lower than the expected ones, aggravating the SNP. The existence of a Solar Neutrino Problem was confirmed also by two radiochemical experiments, studying solar neutrinos via the reaction $\nu_{e}+{ }^{71} \mathrm{Ga} \rightarrow{ }^{71} \mathrm{Ge}+e^{-}$which, thanks to a lower energy threshold $\left(E_{t h}=233 \mathrm{keV}\right)$, made possible the detection also of $p p$ neutrinos. They are the lowest energy and less model-dependent component of neutrino spectra and hence the more robust to test the hypothesis that fusion of hydrogen powers the Sun. Both the soviet-american experiment SAGE, using more than 50 metric tons of metallic gallium [7], and the Gallex experiment at the Gran Sasso underground laboratory, employing 30 metric tons of natural gallium $[8,9$, measured a neutrino signal smaller $(\approx 60 \%)$ than predicted by the SSM. For an historical introduction see for instance [10.

The real breakthrough in solar neutrino physics was the advent of the Sudbury Neutrino Observatory - SNO - experiment. It was able to measure simultaneously, by means of a deuterium Čerenkov detector, three different interaction channels. The neutral current $\nu_{X}+d \rightarrow \nu_{X}+p^{+}+n$, receiving contributions from all active flavors, the elastic scattering $\nu_{X}+e^{-} \rightarrow \nu_{X}+e^{-}$and the charged current $\nu_{e}+d \rightarrow e^{-}+p^{+}+p^{+}$, that is sensitive only to electronic neutrinos. Thank to that it was possible to prove clearly and in a direct way that the measured total neutrino flux was in very good agreement with the SSM predictions, but only a fraction of these neutrinos had conserved their flavor during their travel from the core of the Sun to the Earth. The first SNO data [11, published in 2001, confirmed the results obtained by the other solar neutrino experiments, providing a significant evidence of the presence of a non-electronic active neutrino component in the solar flux. This result indicated, for the first time, the Large Mixing Angle (LMA) as the preferred solution of the SNP, even if different alternative possibilities (LOW-solution, for instance) were still surviving. The SNO Collaboration measured also the neutral current channel, using different techniques. The data collected with these techniques are usually reported as SNO I [12], SNO II [13, with the addition of salt to improve the efficiency of neutral current detection, and SNO III [14], with the use of helium chamber proportional counters.

A fundamental contribution to the solution of the SNP came also from KamLAND. All the previous reactor experiments, using neutrino energy beams of the order of the $\mathrm{MeV}$ with a baseline of the order of $1 \mathrm{~km}$, could test only values of $\Delta m^{2}$ above $10^{-3} \mathrm{eV}^{2}$. The KamLAND experiment, with an average baseline of about $180 \mathrm{~km}$, was, instead, ideal to probe the LMA region, which corresponds to values of $\Delta \mathrm{m}^{2}$ of the order [15, 16, $10^{-5}-10^{-4} \mathrm{eV}^{2}$. The deficit of events observed by the KamLAND Collaboration was inconsistent with the expected rate in absence of oscillation at the $99.95 \%$ confidence level.x The LMA solution was the only oscillation solution compatible with KamLAND results and CPT invariance. Furthermore KamLAND data also restricted the allowed LMA region; the preferred values for $\Delta m_{12}^{2}$ and $\theta_{12}$ are slightly higher than the ones corresponding to the best fit solution of the solar neutrino experiments, but this small difference is explained taking into account the experimental uncertainties. 


\section{Last decade solar neutrino measurements and KamLAND results}

In the second phase of SNO, which began in July 2001, the addition of $2000 \mathrm{~kg}$ of $\mathrm{NaCl}$ to the 1000 metric tons of $D_{2} O$ increased by a factor of 3 the efficiency with respect to the pure $\mathrm{D}_{2} \mathrm{O}$ phase in the detection of neutrons produced in the neutral current $(\mathrm{NC})$ disintegration of deuterons by solar neutrinos and it enhanced the energy of the $\gamma$-ray coming from neutron capture. In this way it was possible (also thanks to the different event isotropy of the multiple $\gamma$ ray emission produced by the neutron capture on ${ }^{35} \mathrm{Cl}$ with respect to the single electron Cerenkov light emitted in charged current interactions) to separate the neutral from the charged current events without any additional assumption on the neutrino energy spectrum. The results, reported in [17, included the full data collected in 391 live days of the salt phase and were analyzed in terms of the CC spectra, with a threshold starting from $5.5 \mathrm{MeV}$ kinetic energy and $\mathrm{NC}$ and ES integrated fluxes separately for day and night. They confirmed the agreement with the SSM for what concerns the $\operatorname{total}^{8} B$ spectrum, favored even larger values of the solar mixing angle and gave no indications of day night asymmetries in $\mathrm{NC}$ rate (which would have been a signal of oscillations to sterile neutrinos or non standard interactions).

During the SNO III phase, started in November 2004, the neutral current signal neutrons were detected with an array of ${ }^{3} \mathrm{He}$ proportional counters deployed in the $\mathrm{D}_{2} \mathrm{O}$ and looking at the gas ionization induced by neutron capture on ${ }^{3} \mathrm{He}$, in order to reduce the fluxes correlation and to improve the accuracy of the determination of the mixing angle. The measured total ${ }^{8} B$ neutrino flux was in substantial agreement with previous measurements and SSM and the ratio of the flux measured with CC and NC turned out to be $\Phi_{C C} / \Phi_{N C}=0.301 \pm 0.033$.

A joint reanalysis of SNO I and SNO II data, known as LETA (Low Energy Threshold Analysis) [18, with improved calibration and analysis techniques, was performed in order to lower the energy threshold, down to an effective electron kinetic energy of $T_{\text {eff }}=3.5 \mathrm{MeV}$. There was a significant improvement in the determination of the neutrino mixing parameters and in the extraction of the total ${ }^{8} B$ neutrino flux, which was measured to be $\Phi_{N C}=$ $\left(5.14_{-0.20}^{+0.21}\right) \times 10^{6} \mathrm{~cm}^{-2} \mathrm{~s}^{-1}$. The analysis of LETA plus SNO III gave a best fit value compatible with the LOW region of parameter space, but the significance level was very similar to the one of the usual LMA solution. A global fit, including all the solar experiments and the data obtained by KamLAND, essentially confirmed, instead, the previous results 14 for $\Delta m_{12}^{2}$. This analysis made possible a further improvement in the angle determination, giving, in a 3 flavor analysis, the following best fit values [19]: $\tan ^{2} \theta_{12}=0.468_{-0.033}^{+0.042} ; \Delta \mathrm{m}_{12}^{2}=$ $\left(7.59_{-0.21}^{+0.20}\right) \times 10^{-5} \mathrm{eV}^{2} ; \sin ^{2} \theta_{13}=\left(2.00_{-1.63}^{+2.09}\right) \times 10^{-2}$.

In the same years also the Super-Kamiokande Collaboration presented new analyses, which essentially confirmed the previous results of the so called SK-I phase. The SK-I data, corresponding to 1496 live days until July 2001, were relative to Elastic Scattering (ES) above $5 \mathrm{MeV}$ and their analysis showed that 
the day-night asymmetry was consistent with zero within $0.9 \sigma$ and in agreement with the predictions of the LMA solution and there were no signals of spectrum distortion and of anomalous periodic variations of the rate (apart from the expected seasonal variation due to Earth's orbit eccentricity). The following analyses included data of the different experimental phases know as Super-Kamiokande II [20] (from December 2002 to October 2005) and SuperKamiokande III (from July 2006 to August 2008) 21] . These two ph,ases substantially confirmed the SK-I results for what concerns the absence of significant spectral distortion, the total ${ }^{8} B$ measured flux and the day-night asymmetry.

Since September 2008, Super-Kamiokande is running with a new data acquisition system and electronics and this allowed a wider dynamic range in the measured charge. This phase of the experiment, denoted as Super-KamiokandeIV 22, is characterized by an optimization of the selection criteria and in particular by the introduction of a new event selection parameter, the "multiple scattering goodness", which has the effect to reduce the very low energy background events (like the ones from ${ }^{214} \mathrm{Bi}$ ). Thanks to the adoption of this selection criteria in the analysis below $7.5 \mathrm{MeV}$ of SK III and SK IV data it has been possible to reach a better signal to noise ratio. In addition to this, a small mistake in the ${ }^{8} B$ energy spectrum shape (with impact on the flux) has been found by the SK Collaboration and fixed in this new analysis, which includes an updated live time of 1069 days. The kinetic energy threshold has been slightly lowered, down to $3.5 \mathrm{MeV}$, and could be further reduced in the near future. The total systematic error has been reduced to $1.7 \%$ (from the $3.2-3.5 \%$ of SK-I and the $2.1 \%$ of SKIII). The value measured for elastic scattering would correspond, in absence of oscillation, to a ${ }^{8} B$ neutrino flux equal to $(2.34 \pm 0.03($ stat. $) \pm 0.04($ syst. $)) \times 10^{6} \mathrm{~cm}^{-2} \mathrm{~s}^{-1}$. The day-night asymmetry is still consistent with zero within $2.3 \sigma$.

Quite recently the SNO Collaboration performed a combined three phases analysis, in which all the data of the experiment were combined into a single dataset 23. The study took advantage from the use of a pulse shape analysis (making possible a better particle identification) and a signal extraction based on a survival probability fit independent on any flux model. The flux determination extracted from the signal[19], $\Phi_{8_{B}}=\left(5.25 \pm 0.16_{-0.013}^{+0.011}\right) \times 10^{6} \mathrm{~cm}^{-2} \mathrm{~s}^{-1}$, is in good agreement with the SNO-LETA results. The total uncertainty is slightly larger than 3\% and it is independent on the Solar Standard Model and on the oscillation models The oscillation parameter study is also improved with respect to previous analyses 1 . The analysis is presently under revision, to include recent $\theta_{13}$ measurements and the updated results should be published soon. Once more, the SNO contribution is dominant for the $\theta_{12}$ determination, but it is not sufficient by itself to exclude the LOW solution, which is anyhow eliminated by Borexino and KamLAND independently. In addition to the important role played in the determination of ${ }^{8} B$, SNO also aims to improve the study of hep neutrinos, which represent the highest energy and lowest flux contrbution of the solar neutrino spectrum.

\footnotetext{
${ }^{1}$ For the exact determination of the mixing parameter values see [19].
} 
The KamLAND experiment played a fundamental role in solar neutrino physics, starting from its first data [24], that were determinant to prove the validity of the oscillation hypothesis showing that the LMA solution was the correct one. A new set of data were collected between March 2002 and January 2004 with an important upgrade on the detector, in which the photocatode coverage was increased and the energy resolution improved. Furthermore an improved analysis, with a background reduction made possible by better techniques in the event selection cuts based on the time, position and geometry of the events, was performed. The study included the data obtained in the 2002-2004 campaign and a re-analysis of data obtained before. The observed events above $2.6 \mathrm{MeV}$ were 258 , against an expected number of antineutrino events in absence of antineutrino disappearance equal to $365.2 \pm 23.7$ (syst); this corresponds to a $\bar{\nu}_{e}$ survival probability equal to $0.658 \pm 0.044$ (stat) \pm 0.047 (syst). The best fit obtained from this analysis was in the so-called LMAI region with values of $\Delta m_{12}^{2}$ around $8 \cdot 10^{-5} \mathrm{eV}^{2}$. Including in the KamLAND analysis the results coming from the solar neutrino experiments, the allowed values of the angle were restricted and the two flavor combined analysis gave $\Delta m_{12}^{2}=7.9_{-0.5}^{+0.6} \times 10^{-5} \mathrm{eV}^{2}$ , $\tan ^{2} \theta_{12}=0.40_{-0.07}^{+0.10}$ at a $1 \sigma$ level [25].

The following KamLAND analysis included new data collected up to May 2007 [26] ; it was obtained with a further reduction of systematic uncertainties and of background. Furthermore the radius of the fiducial volume was increased from 5.5 to $6 \mathrm{~m}$. The allowed oscillation parameter values were $\Delta m_{12}^{2}=$ $\left(7.58_{-0.13}^{+0.14}(\text { stat })_{-0.15}^{+0.15}(\right.$ syst $\left.)\right) \times 10^{-5} \mathrm{eV}^{2}$ and $\tan ^{2} \theta_{12}=0.56_{-0.07}^{+0.10}(\text { stat })_{-0.06}^{+0.10}($ syst $)$, for $\tan ^{2} \theta_{12}<1$. In the three neutrino oscillation analysis the main effect was to enlarge the uncertainty on $\theta_{12}$, leaving $\Delta m_{12}^{2}$ substantially unchanged.

In the last five years a new important step forward was made possible by Borexino Collaboration, which started the data taking in May 2007. Borexino, thank to the ability to reach a very low radiopurity level at the ton scale [27, is the first real time experiment investigating the low and medium energy part of solar neutrino spectrum.

One of its main focus is the study of the ${ }^{7} \mathrm{Be}$ monochromatic line. In the first analysis performed by the Collaboration the ${ }^{7} \mathrm{Be}$ signal was extracted from the background using data collected in 47.4 live days. The best value estimate for the rate was $47 \pm 7$ (stat) \pm 12 (syst) counts/(day $\cdot 100$ ton), where the systematic error was mainly due to the fiducial mass determination [28]. A second release of data was reported after 9 months from an analysis of 192 live days, corresponding to 41.3 ton-yr fiducial exposure to solar neutrinos. The total estimated systematic error was 29 8.5\%. The best value for the interaction rate of the $0.862 \mathrm{MeV}^{7} \mathrm{Be}$ solar neutrinos was $49 \pm 3$ (stat) \pm 4 (syst) counts/(day·100 ton), in very good agreement with the predictions of the LMA oscillation solution: $48 \pm 4$ counts/(day·100 ton).

In order to reduce the systematic uncertainties a calibration campaign was performed in 2009 introducing inside the detector several internal radioactive sources $\alpha$ 's, $\beta$ 's, $\gamma$ 's, and neutrons, at different energies and in hundreds of different positions. Thanks to this calibration campaign, the systematic error was 
reduced to $2.7 \%$ and the total uncertainty to $4.3 \%$. The data set run from May 2007 to May 2010, with a fiducial exposure equivalent to 153.6 ton $\cdot y e a r$. The ${ }^{7} B e$ solar neutrino rate was evaluated to be $46.0 \pm 1.5$ (stat) \pm 1.3 (syst) counts/(day·100 ton) [30] .

Another important contribution of Borexino to the knowledge of solar neutrinos has been the measurement of the fluxes of pep and CNO neutrinos, that had never been directly measured before. These measurements are important because in the Solar Standard Models the flux of pep neutrinos is predicted with a small uncertainty (around 1\%), due to the solar luminosity constraint and to their direct connection to $p p$ neutrinos; therefore its experimental determination would be a stringent test of the validity of these astrophysical models. On the other hand, the detection of neutrinos within the $C N O$ bi-cycle is central to probe the solar core metallicity and contribute in this way to the solution of the solar metallicity problem [31, 32] .

The expected rate from pep and $C N O$ neutrino interaction is on the order of a few counts per day in a 100 ton target. In order to detect pep and $C N O$ neutrinos the Borexino Collaboration adopted a novel analysis procedure to suppress the main source of background in the $1-2 \mathrm{MeV}$ energy range, which is due to the cosmogenic $\beta^{+}$-emitter ${ }^{11} \mathrm{C}$ (lifetime of $29.4 \mathrm{~min}$ ) produced within the scintillator by muon interactions with ${ }^{12} \mathrm{C}$ nuclei. The background due to ${ }^{11} \mathrm{C}$ can be reduced by performing a space and time veto following coincidences between signals from the muons and the cosmogenic neutrons 33. This technique (Three-Fold Coincidence, TFC) is based on the reconstructed track of the muon and the reconstructed position of the neutron-capture $\gamma$-ray. This criterium of rejection gave a rate of $(2.5 \pm 0.3)$ counts per day due to muons, corresponding to about $(9 \pm 1) \%$ of the original rate, while preserving $48.5 \%$ of the initial exposure. Thanks to a small difference in the time distribution of the scintillation signal that arises from the finite lifetime of ortho-positronium as well as from the annihilation $\gamma$-rays, which present a distributed, multi-site event topology and a larger average ionization density than electron interactions, it is possible to discriminate ${ }^{11} \mathrm{C} \beta^{+}$decays from neutrino-induced $e^{-}$recoils and $\beta^{-}$decays exploiting the pulse shape differences between $e^{-}$and $e^{+}$interactions in organic liquid scintillators.

The Borexino Collaboration presented the results in an analysis, published in 2012 34, based on a binned likelihood multivariate fit performed on pulse shape, spatial distributions and energy. In the energy region of interest a fit procedure was applied to radioactive backgrounds and to the contribution from $p p$ solar neutrinos, that was fixed to the SSM assuming a MSW-LMA value of 35 $\tan ^{2} \theta_{12}=0.47_{-0.04}^{+0.05}, \Delta m_{12}^{2}=(7.6 \pm 0.2) \times 10^{-5} \mathrm{eV}^{2}$, and to the contribution from ${ }^{8} \mathrm{~B}$ neutrinos to the rate from the measured flux of LETA and SNOI+II+III. The obtained results for the pep and CNO neutrino interaction rates, in units of counts/(day.100ton), are $3.1 \pm 0.6_{\text {stat }} \pm 0.3_{\text {syst }}$ and $<7.9$ $\left(<7.1_{\text {stat only }}\right)$ respectively, corresponding to a solar- $\nu$ flux of $(1.6 \pm 0.3) \times$ $10^{8} \mathrm{~cm}^{-2} \mathrm{~s}^{-1}$ for pep and $<7.7 \times 10^{8} \mathrm{~cm}^{-2} \mathrm{~s}^{-1}$ for $C N O$. 


\section{The mass and mixing pattern}

In many cases the solar neutrino data global analysis have been performed in the two flavor approximation. This simplifying assumption was justified by the fact that the upper limit derived by the reactor experiments for the mixing angle between the first and the third mass generation was quite severe and, therefore, the results of the analysis done with $\theta_{13}=0$ were presumably a good approximation. A recent example can be found, for instance, in 23]. In that case, as already reported in section (3), the main results obtained by SNO collaboration also in the two flavor analysis were the following: the SNO data are fundamental to determine the value of the $\theta_{12}$ mixing angle, but it is essential to include also the results of the previous solar neutrino experiments and of Borexino and the ones from KamLAND to select definitely the LMA solution and to restrict significantly the allowed values of $\Delta m_{12}^{2}$. The small tension emerging between the solar neutrino results and the KamLAND data (that would prefer slightly larger values for $\Delta m_{12}^{2}$ ) is reduced in the full three flavor analysis. The best fit is obtained for values of $\theta_{13}$ different from zero $\left(\sin ^{2} \theta_{13}=\right.$ $0.025_{-0.015}^{+0.018}$ ) and the corresponding selected regions in the 12 mixing parameter plane are [19] : $\tan ^{2} \theta_{12}=0.446_{-0.036}^{+0.048} ; \Delta m_{12}^{2}=\left(7.41_{-0.19}^{+0.21}\right) \times 10^{-5} \mathrm{eV}^{2}$.

The indication in favor of values of $\theta_{13}$ different from zero was in agreement also with the outcome of recent theoretical analyses [36 and was strongly confirmed during this year by a series of results from the long baseline experiments T2K [37] and MINOS [38] and, above all, by three different neutrino reactor experiments [39, 40, 41], which found values of $\sin ^{2} \theta_{13}$ centered between 0.020 and 0.030 .

Both in two and three flavor analyses, the higher values of $\Delta m_{12}^{2}$ in the LMA region were excluded, together with the full LOW solution, mainly thanks to the large discrimination power of KamLAND. This experiment, however, did not contribute significantly to improve the mixing angle determination and the uncertainty on this parameter remained quite high. For a comparison between the results obtained in the two and in the three flavor analyses one can look for instance at the Tables IX and X of [23, in which the results of the recent analysis of all the solar + KamLAND results, performed by the SNO collaboration, are summarized.

As already said, the precise determination of the ${ }^{8} B$ solar neutrino flux, $\Phi_{8_{B}}=\left(5.25 \pm 0.16\right.$ (stat) ${ }_{-0.013}^{+0.011}$ (syst) $) \times 10^{6} \mathrm{~cm}^{-2} \mathrm{~s}^{-1}$, made possible by the combined analysis of the different SNO phases, presents a significant reduction of the systematic uncertainty. This result was consistent with, but more precise than, both the high-Z BPS09(GS), $\Phi=(5.88 \pm 0.65) \times 10^{6} \mathrm{~cm}^{-2} \mathrm{~s}^{-1}$, and low-Z BPS09(AGSS09), $\Phi=(4.85 \pm 0.58) \times 10^{6} \mathrm{~cm}^{-2} \mathrm{~s}^{-1}$, solar model predictions [42]

Recently different groups published the results of global phenomenological studies of the mass and mixing parameters (see [43, 44, 45, 46]), finding a substantial agreement. We refer the interested reader to these papers for a detailed analysis of mixing parameters and for a discussion about the promising perspectives for leptonic CP violation searches opened by the interval of allowed values for $\sin ^{2} \theta_{13}$ selected by the recent experimental results [43. 
The combination of the recent SNO collaboration's analyses [18, 23] and of the Borexino measurements [47] made possible a detailed study of the low energy part of the ${ }^{8} B$ solar neutrino spectrum. Even if characterized by a larger uncertainty (mainly due to a more limited statistics), Borexino data confirm the LETA indication of low energy data points lower than the theoretical expectations based on matter enhanced oscillation and solar models. These results were in agreement also with the Super-Kamiokande observation of a flat spectrum, consistent with the undistorted spectrum hypothesis. The emergence of this slight tension between theory and experiments seems to indicate the presence of new subdominant effects and also suggests the possibility of non-standard neutrino interactions (like those studied in [48]) or the mixing with a very light sterile neutrino 49, 46. Future solar neutrino experiments, like $\mathrm{SNO}+$, could shed more light on this subject, by performing precision measurements of lower energies solar neutrinos (like the pep neutrinos).

The accuracy reached by the data obtained at different solar neutrino experiments suggests also the possibility of using these results to test in an independent way the neutrino propagation and mixing models from one side and the astrophysical models ruling neutrino production on the other side. With this aim it is possible to perform a so called "free flux analysis", in which one lets the different solar neutrino fluxes vary in order to accommodate the experimental data, maintaining the functional dependences as predicted by the standard models and assuming a "luminosity constraint" (which assures that the fusion processes are responsible for solar luminosity and guarantees the conservation of energy for nuclear fusion of light elements). The main aspects and the results of such an analysis are discussed for instance in 50 . The precision of the ${ }^{7} \mathrm{Be}$ and ${ }^{8} \mathrm{~B}$ neutrino fluxes is driven by the Borexino and SNO (SK) neutrino experiments, while the precision of the $p p$ and pep neutrino fluxes at present mainly comes by the imposition of the luminosity constraint. The neutrino data directly demonstrates that the Sun shines by the $p p$-chain and the $C N O$ bi-cycle only contributes to the total luminosity at the percent level (around $0.8 \%$ and $0.4 \%$, respectively for $h i g h-Z$ and low- $Z$ solar models). If one further relaxes the assumption, giving up the luminosity constraint, one gets an estimate of solar luminosity inferred by neutrino data which is in agreement with the directly measured one within about $15 \%$.

\section{Future of solar neutrino physics}

In the near future the study of solar neutrinos, for what concerns the pp-chain, will be focused on the low energy part, which represents the great majority of the spectrum, but up to now has been an almost unexplored realm. A significant contribution is expected from Borexino and $\mathrm{SNO}+$ [51] experiments. The Borexino Collaboration already proved its capability to perform the first measurements of pep and CNO neutrinos. Since July 2010 a big effort was undertaken, with a new purification campaign, in order to further reduce the main radioactive background sources. Being SNOLAB located twice deeper un- 
derground than the Gran Sasso laboratory, the SNO+ experiment, that should start taking data soon, will take advantage from a lower muon flux and hence a strongly reduced ${ }^{11} \mathrm{C}$ rate. This could determine a fundamental improvement in the pep neutrino measurement, where a $5 \%$ uncertainty is expected. Both collaborations hope to attack the main problem of measuring the lowest energy parts of the solar neutrino spectrum, the $p p$ neutrinos and the $0.38 \mathrm{MeV}$ Beryillium line, even if the presence of ${ }^{14} \mathrm{C}$ background in the organic scintillators will make this very low energy measurements a very hard task.

The pile up effects of ${ }^{14} \mathrm{C}$ are being studied with Monte Carlo simulations and using the data themselves. The Borexino Collaboration is planning to design also a possible test of these pile-up events by inserting $\mathrm{CO}_{2}$, in gaseous form, in the scintillator. This would increase of a factor of about 2 the contribution from ${ }^{14} \mathrm{C}$ and then the pile up induced from it. In this way, comparing the data before and after the $\mathrm{CO}_{2}$ insertion, it could be easier to disentangle the ${ }^{14} \mathrm{C}$ pile up effects.

All future experiments aiming to measure the low energy part of solar neutrino spectrum are characterized by a very large detector target mass and by the need to reach very high radiopurity levels, in order to be suitable for the detection of a low rate signal in a region characterized by different potential radioactive background sources. This requirement is common also to the experiments looking for neutrinoless double $\beta$ decay or for dark matter signals (search for signatures of WIMPs) and, therefore, many of the planned solar neutrino experiments are multipurpose experiments designed also for the other above-quoted topics.

Thanks to the experience acquired with Borexino, a new generation of neutrino detectors has been proposed. LENA (Low Energy Neutrino Astronomy) [52] is a multipurpose detector aiming to study, among others issues, solar neutrinos. The project consists in a cylindrical detector with a diameter of $30 \mathrm{~m}$ and a length of about $100 \mathrm{~m}$ for about 50 kilotons of liquid scintillator as target mass. The light is collected by about $45,000,20 \mathrm{~cm}$ in diameter, photomultipliers equipped with conic mirrors. The corresponding surface coverage is about $30 \%$ and the solvent for the liquid scintillator will probably be linear alkylbenzene which has a high light yield and large attenuation length on the order of 10 to $20 \mathrm{~m}$ at a wavelength of $430 \mathrm{~nm}$. The photoelectron yield is about 200 photoelectrons per $\mathrm{MeV}$ for a scintillator mixture containing $2 \mathrm{~g} / \mathrm{l} \mathrm{PPO}$ and $20 \mathrm{mg} / \mathrm{l}$ bisMSB as wavelength shifters. As alternative solvent option LENA could employ the PXE [53] or a mixture of PXE and dodecane. A high statistics can be reached in short times and in both Pyhsalmi and Frejus underground laboratories where the muon flux is lower compared to LNGS underground laboratories. For pep, CNO and low-energy ${ }^{8} \mathrm{~B}-\nu \mathrm{s}$ detection a fiducial mass of $\sim 30 \mathrm{kton}$ is mandatory, while the fiducial mass for ${ }^{7} \mathrm{Be}-\nu$ s and high-energy $(E>5 \mathrm{MeV})$ ${ }^{8} \mathrm{~B}-\nu \mathrm{s}$ could be enlarged to $35 \mathrm{kton}$ or more. The expected rates (for the channel $\nu e \rightarrow e \nu$ ) in 30 kton for $p p$ neutrinos, pep neutrinos and the $C N O$ bi-cycle, using the most recent solar model predictions are $40 \mathrm{cpd}$ (counts per day), 280 cpd and $190 \mathrm{cpd}$ respectively; while for ${ }^{7} \mathrm{Be}-\nu \mathrm{s}$ and ${ }^{8} \mathrm{~B}-\nu$ s they are $100 \mathrm{cpd}$ and $79 \mathrm{cpd}$ in 35 kton fiducial mass [52] . 
The Low Energy Neutrino Spectroscopy (LENS) detector has as main goal the real time measurement, as a function of their energy, of solar neutrinos and particularly of the $p p$ ones. To make an energy spectrum measurement on low energy neutrinos, one has to reach a low threshold for the charged current (CC) process and discriminate the radioactive background. The $\mathrm{CC}$ process employed in LENS is the neutrino induced transition of ${ }^{115} \mathrm{In}$ to an excited state of ${ }^{115} \mathrm{Sn}$ (i.e. $\left.\nu_{e}+{ }^{115} \mathrm{In} \rightarrow{ }^{115} \mathrm{Sn}^{*}+e^{-}\left(\mathrm{E}=\mathrm{E}_{\nu}-114 \mathrm{keV}\right)\right)$ followed by ${ }^{115} \mathrm{Sn}^{*}(\tau=4.76 \mu s) \rightarrow{ }^{115} \mathrm{Sn}+\gamma(498 \mathrm{keV})+\gamma(116 \mathrm{keV})$. Thanks to this reaction it is possible to detect low energy neutrinos with a threshold of $114 \mathrm{keV}$ and measure their energy. The primary interaction and the secondary cascade give a triple coincidence, correlated in time and space. The detection medium is a liquid scintillator chemically doped with natural indium $\left({ }^{115} \mathrm{In}=95.7 \%\right)$. LENS should be able to determine the low energy solar neutrino fluxes with an accuracy $\leq 4 \%$ [54.

Another interesting possibility, under investigation by different collaborations, is that of using scintillation detectors with liquid noble gases, like xenon, argon and neon. These materials are relatively inexpensive, easy to obtain and dense; moreover, they can be quite easily purified, have a high scintillation yield (about $30-40$ photons $/ \mathrm{keV}$ ) and do not absorb their own scintillation light.

A first example is given by the CLEAN/DEAP family, a kind of detectors based entirely on scintillation in liquid neon (LNe) and liquid argon (LAr). Some prototypes of this kind have been installed in the SNOLAB. The CLEAN (Cryogenic Low Energy Astrophysics with Noble gases) detector [55. will be made by a stainless steel tank, of about 6 meters of diameter, filled with 135 metric tons of cryogenic liquid neon; only the central part of it, surrounded isotropically by a series of photomultipliers, will constitute the detector fiducial volume. An external water tank will act as $\gamma$-ray and neutron shielding and muon veto. A statistical uncertainty on the $p p$ measurements of the order of $1 \%$ is foreseen.

The XMASS experiment [56] is a multipurpose experiment, mainly focused on neutrinoless double $\beta$ decay and dark matter searches, but designed also to study $p p$ and ${ }^{7} B e$ neutrinos. It will employ liquid xenon and should reach very low levels of background and energy threshold. The full XMASS detector will have a fiducial volume of 10 metric tons.

Another experimental project based on the noble gases liquid scintillator technique is that of DARWIN (DARk matter WImp search with Noble liquids) [57, conceived for the study for a future multi-ton scale LAr and LXe dark matter search facility in Europe. The main goal of the experiment is to look for a WIMP signal, but the energy region of the nuclear recoil spectrum, below $200 \mathrm{keV}$, that should be investigated by this future experiment is of particular interest also for the study of the $p p$ solar neutrinos.

\section{Conclusions}

In the last 10-15 years very important steps forward have been done in the study of solar neutrinos and consequently in our knowledge of neutrino properties. The 
results obtained around the turn of the century by the Cerenkov experiments SuperKamiokande and SNO and the confirmation offered by the KamLAND experiment monitoring the reactor antineutrinos were determinant to solve the long standing Solar Neutrino Puzzle, proving in a crystal clear way the validity of the oscillation hypothesis and selecting the Large Mixing Angle solution in the mixing parameter space. In the following years the aforementioned experiments went on producing data improving their statistics, reducing the systematic errors with the introduction of new detection techniques (for instance in the case of the neutral current studies performed by the SNO collaboration) and improved methods of statistical analysis and in some cases lowering their energy threshold. In the last five years a new piece of the puzzle was added, thanks to Borexino experiment, which performed the first real time measurement of the low energy part of solar neutrino spectrum, observing with increasing accuracy the monochromatic beryllium line and obtaining the first measurements of pep and $C N O$ neutrinos. Meanwhile a long standing question found its answer during the last year with the discovery at different long baseline reactor experiments that the $\theta_{13}$ mixing angle is definitely different from zero.

A generally coherent picture emerges from all of these experimental achievements and from the global analyses performed by the single experimental collaborations and in other theoretical and phenomenological studies and the basic properties and the values of the parameters driving solar neutrino oscillations, $\Delta m_{12}^{2}$ and $\theta_{12}$, are now known with quite a satisfactory accuracy. Nevertheless, there are still important aspects of the oscillation mechanism on which it would be desirable to shed more light. In particular the transition between the low energy region, where the oscillation takes place essentially in vacuum, and the higher energy regime, in which matter interactions become extremely relevant, requires more study. In fact the data available up to now for the low energetic ${ }^{8} B$ neutrinos (which are affected by a significant uncertainty) don't show explicitly the rise of the Solar neutrino spectrum 58 that, according to the LMA solution, should appear when one decreases the energy passing from the matter dominated towards the vacuum region. More data coming from SuperKamiokande, Borexino and SNO+ experiments are expected to further explore the conversion in this regime. The precise measurement of low energy neutrinos like $p e p$ (and the comparison with eventual future $p p$ neutrino measurement), exploiting the fact that pep neutrinos are more energetic than the ${ }^{7} B e$ ones, could also help to see small solar matter effects in the flavor conversion. The $C N O$ neutrinos would be even more suitable for this kind of studies, because their energy is of the same order of the pep ones, but they are produced at higher temperatures and therefore at higher densities, leading to even more significant matter effects.

The combination of all of these studies and of the results by reactor experiments is expected to eliminate in the near future this uncertainty, clarifying if the oscillation mechanism is well understood or one has to invoke the so called non standard interactions.

From an astrophysical point of view, the accuracy reached by the solar neutrino experiments is for many aspects sufficient to realize the original Davis and 
Bahcall's dream and use neutrinos as probes to get a better insight into the mechanisms driving the fusion processes in the Sun. Generally speaking the Standard Solar Model predictions are in excellent agreement with solar fluxes, regardless of the solar composition assumed in the construction of the model. However the measured values of the fluxes for the different components of the $p p$-fusion chain unfortunately fall somehow in the middle 31 between the predicted values for the two different versions of the solar models usually denoted as high- $Z$ and low- $Z$ solar models and, therefore, it is very unlikely that the study of neutrino fluxes from the $p p$-chain alone will be able to discriminate between these different solar compositions. An important possibility could be offered by the combined analysis of the different components of pp-chain and of CNO neutrinos (which can bring important information about the solar core). Borexino recently opened a new way, establishing the most stringent upper limit on $C N O$ fluxes which is about a factor of 1.5 larger than solar model predictions and the recent developments, also in background subtraction techniques, offer to this experiment the possibility of a further improvement. In this regard, the potentialities of $\mathrm{SNO}+$ are even more promising, thanks to its location deeper underground and to the higher detector mass, but at present the primary solar neutrino measurements are planned to take place after the double beta decay measurements are carried out for at least 4 years.

This issue is relevant because it could make possible an important step forward in the solution of the so called solar metallicity problem 31, 32. In the last decades three-dimensional radiation hydrodynamic (3D RHD) models of the solar atmosphere have been consistently developed by different groups 59 . 60 61. Their main impact on solar models and solar neutrino physics is that the composition of different elements have been significantly reduced (down to $30-40 \%$ for some key elements, like C, N and O). However these models, usually denoted as low- $Z$ models, seem to be definitely less efficient than previous high- $Z$ models in matching helioseismic constraint, like the internal sound speed and density profiles and the depth of the solar convective envelope. The different attempts developed in these years to solve this solar abundance, or solar metallicity, problem by modifying some inputs of the Solar Standard Models partially failed, in the sense that a simultaneous solution to all the problems of consistency with helioseismology has not yet been found. A possible way out could be offered by an increase of radiative opacities, but the increase of this factor needed to restore the agreement with helioseismology seems be much higher than the estimated present uncertainty of its present calculations in the low- $Z$ models. It's worth noticing that the ${ }^{7} B e$ and ${ }^{8} B$ fluxes of low- $Z$ SSMs with increased opacity would essentially coincide with those from a high- $Z$ model 62, showing the intrinsic degeneracy between composition and opacities. By using ${ }^{8} \mathrm{~B}$ and now ${ }^{7} \mathrm{Be}$ as thermometers of the solar core [63], $\mathrm{CNO}$ neutrinos represent a unique way to break this degeneracy and provide an independent determination of the $C N O$ abundances, particularly the $\mathrm{C}+\mathrm{N}$ abundance in the solar core. Keeping in mind the antagonism between solar interior and solar atmosphere models that the solar abundance problem has established, results from $C N O$ fluxes will be of the outmost relevance for solar, and by extension 
stellar, physics.

A precise measurement of $C N O$ neutrinos would have a great impact on our knowledge of the formation and evolution mechanism not only of the Sun, but also of other stars and of the planets in the solar system. First of all the detection of these neutrinos would confirm directly that the $C N O$ bi-cycle is an important source of energy for the stars (which can become the dominant fusion mode for stars with masses right above the solar one). Moreover, an accurate determination of the combined ${ }^{13} N+{ }^{15} \mathrm{O}$ flux and consequently of the solar $C+N$ abundance would be extremely useful not only for the solution of the solar abundance problem, but also for a better knowledge of the mixing

mechanism involved in the evolution of the Sun and in the earlier phases of planet formation. For a more detailed discussion of this topic we refer the interested reader to [50].

\section{Acknowledgements}

It's a pleasure for us to thank A. M. Serenelli and C. Peña Garay, who realized with us the paper [50], on which this review is partially based.

\section{References}

[1] J. N. Bahcall, Phys. Rev. Lett. 12 (1964) 300.

[2] R. J. Davis, Phys. Rev. Lett. 12 (1964) 302.

[3] R. J. Davis, D. S. Harmer and K. C. Hoffman.

[4] Phys. Rev. Lett. 20 (1968) 1205.

[5] K. S. Hirata et al., Phys. Rev. Lett. 63 (1989) 16.

[6] Y. Fukuda et al., Phys. Rev. Lett. 82 (1999) 2430.

[7] J.N. Abdurashitov et al., Phys. Rev. Lett. 83 (1999) 4686.

[8] W. Hampel et al., Phys. Lett. B 447 (1999) 127.

[9] M. Altmann et al., Phys. Lett. B 616 (2005) 174.

[10] L. Miramonti and F. Reseghetti, Riv. Nuovo Cim. 25N7 (2002) 1.

[11] Q. R. Ahmad et al., Phys. Rev. Lett. 87 (2001) 071301.

[12] Q. R. Ahmad et al., Phys. Rev. Lett. 89 (2002) 011301.

[13] S. N. Ahmed et al., Phys. Rev. Lett. 92 (2004) 181301.

[14] B. Aharmim et al., Phys. Rev. Lett. 101 (2008) 111301.

[15] A. Piepke, Nucl. Phys. Proc. Suppl. 91 (2001) 99. 
[16] P. Aliani, V. Antonelli, M.Picariello and E. Torrente-Lujan, New J. Phys. 5 (2003) 2.

[17] B. Aharmim et al., Phys. Rev. C 72 (2005) 055502.

[18] B. Aharmim et al., Phys. Rev. C 81 (2010) 055504.

[19] N. Fiuza de Barros, "Final Results from SNO", talk given at the "Physun 2012" conference, LNGS October 2012, available at the conference site.

[20] J. P. Cravens et al., Phys. Rev. D 78 (2008) 032002.

[21] K. Abe et al., Phys. Rev. D 83 (2011) 052010.

[22] M. Smy, arXiv:1110.0012 See also the talk Recent results of SuperKamiokande, given by Y. Takeushi at the "Physun 2012" conference, LNGS October 2012.

[23] B. Aharmim et al., arXiv:1109.0763 [nucl-ex].

[24] K. Eguchi et al., Phys. Rev. Letts. 90 (2003) 021802.

[25] T. Araki et al., Phys. Rev. Lett. 94 (2005) 081801.

[26] S. Abe et al., Phys. Rev. Lett. 100 (2008) 221803.

[27] C. Arpesella et al., Astropart. Phys. 18 (2002) 1.

[28] C. Arpesella et al., Phys. Lett. B 658 (2008) 101.

[29] C. Arpesella et al., Phys. Rev. Lett. 101 (2008) 091302.

[30] G. Bellini et al., Phys. Rev. Lett. 107, 141302 (2011).

[31] A. M. Serenelli, W. C. Haxton and C. Peña-Garay, Astrophys. J. 743 (2011) 24.

[32] S. Basu, "Astronomical Society of the Pacific Conference Series", vol. 416 (2009) 193.

[33] H. Back et al., Phys. Rev. C 74 (2006) 045805.

[34] G. Bellini et al., Phys. Rev. Lett. 108 (2012) 051302.

[35] Review of Particle Physics, K. Nakamura et al., J. Phys. G 37 (2010) 075021.

[36] G. L. Fogli, E. Lisi, A. Marrone, A. Palazzo and A. M. Rotunno, Phys. Rev. D 84 (2011) 053007.

[37] K. Abe et al., Phys. Rev. Lett. 107 (2011) 041801.

[38] P. Adamson et al., Phys. Rev. Lett. 107 (2011) 181802. 
[39] Y. Abe et al., Phys. Rev. Lett. 108 (2012) 131801.

[40] F. P. An et al., Phys. Rev. Lett. 108 (2012) 171803.

[41] J. K. Ahn et al., Phys. Rev. Lett. 108 (2012) 191802.

[42] A. M. Serenelli, S. Basu,J. Ferguson, M.Asplund, Astrophys. J. Lett. 705 (2009) L123.

[43] G. L. Fogli, E. Lisi, A. Marrone, D. Montanino, A. Palazzo and A. M. Rotunno, Phys. Rev. D 86 (2012) 013012.

[44] D. V. Forero, M. Tortola and J. W. F. Valle, Phys. Rev. D 86 (2012) 073012 .

[45] M.C. Gonzalez-Garcia, M. Maltoni, J.Salvado and T.Schwetz, JHEP 1212 (2012) 123.

[46] A. Y. .Smirnov, arXiv:1210.4061 [hep-ph].

[47] G. Bellini et al., Phys. Rev. D 82 (2010) 033006.

[48] A. Friedland, C. Lunardini and C. Peña-Garay, Phys. Lett. B 594 (2004) 347.

[49] P. C. de Holanda and A. Y. .Smirnov, Phys. Rev. D 83 (2011) 113011.

[50] V. Antonelli, L. Miramonti, C. Peña-Garay, and A. Serenelli, Advances in High Energy Physics, Vol. 2012 (2012), Article ID 351926.

[51] C. Kraus et al., Prog. Part. Nucl. Phys. 64 (2010) 273.

[52] M. Wurm et al., Astropart. Phys. 35 (2012) 685.

[53] H. Back et al., Nucl. Instrum. and Methods in Physics Research A 585 (2008) 4860.

[54] R. S. Raghavan, "Discovery potential of low energy solar neutrino experiments", Notes for APS-SAWG, Mar 152004.

[55] D. N. McKinsey and K. J. Coakley, Astropart. Phys. 22 (2005) 355.

[56] S. Moriyama, PoS IDM 2010 (2011) 057.

[57] L. Baudis, PoS IDM 2010 (2011) 122. L. Baudis, arXiv:1201.2402.

[58] A. Chavarria, arXiv:1201.6311 [astro-ph.SR].

[59] M. Asplund, N. Grevesse, A. J. Sauval, P. Scott, Ann. Rev. Astron. Astrophys. 47 (2009) 481.

[60] M. Asplund, N. Grevesse and J. Sauval, Nucl. Phys. A 777 (2006) 1. 
[61] B. Beeck, R. Collet, M. Steffen, M. Asplund, R. H. Cameron, B. Freytag, W. Hayek and H. G. Ludwig et al., Astronom. and Astrophys. vol. 539 (2012) article 121.

[62] A. M. Serenelli, Astrophysics and Space Science, vol. 328, pp.13, 2010.

[63] W. C. Haxton and A. M. Serenelli, Astrophys. J. 687 (2008) 678. 